\title{
Long term outcome after transjugular intrahepatic portosystemic stent-shunt in non-transplant cirrhotics with hepatorenal syndrome: a phase II study
}

\author{
K A Brensing, J Textor, J Perz, P Schiedermaier, P Raab, H Strunk, H U Klehr,
} H J Kramer, U Spengler, H Schild, T Sauerbruch

\begin{abstract}
Background-Recent small studies on hepatorenal syndrome (HRS) indicate some clinical benefit after transjugular intrahepatic portosystemic stent-shunt (TIPS) but sufficient long term data are lacking.

Aim-We studied prospectively feasibility, safety, and long term survival after TIPS in 41 non-transplantable cirrhotics with HRS (phase II study).

Patients and methods-HRS was diagnosed using current criteria (severe (type I) HRS, n=21; moderate (type II) HRS, $n=20$ ). Thirty one patients (14 type I, 17 type II) received TIPS $(8-10 \mathrm{~mm})$ while advanced liver failure excluded shunting in 10. During follow up (median 24 months) we analysed renal function and survival (Kaplan-Meier).

Results-TIPS markedly reduced the portal pressure gradient (21 (5) to 13 (4) mm Hg (mean (SD)); p<0.001) with one procedure related death $(3.2 \%)$. Renal function deteriorated without TIPS but improved $(p<0.001)$ within two weeks after TIPS (creatinine clearance 18 (15) to 48 (42) $\mathrm{ml} / \mathrm{min}$; sodium excretion 9 (16) to 77 (78) $\mathrm{mmol} / 24$ hours) and stabilised thereafter. Following TIPS, three, six, 12, and 18 month survival rates were $81 \%, 71 \%$, $48 \%$, and $35 \%$, respectively. As only $10 \%$ of non-shunted patients survived three months, total survival rates were $63 \%$, $56 \%, 39 \%$, and $29 \%$, respectively. Multivariate Cox regression analysis revealed bilirubin $(p<0.001)$ and HRS type $(p<0.05)$ as independent survival predictors after TIPS.

Conclusions-TIPS provides long term renal function and probably survival benefits in the majority of non-transplantable cirrhotics with HRS. These data warrant controlled trials evaluating TIPS in the management of HRS.

(Gut 2000;47:288-295)
\end{abstract}

Keywords: hepatorenal syndrome; transjugular intrahepatic portosystemic stent-shunt; liver cirrhosis; portal hypertension; ascites; renal failure

Hepatorenal syndrome (HRS) is a common and severe complication of advanced liver cirrhosis. After the onset of HRS, patients have only a minimal chance of renal functional recovery and a poor prognosis under medical therapy. Nine out of 10 patients with advanced HRS die within 10 weeks, most within the first month after diagnosis. ${ }^{1}$ To date, the only established therapy that guarantees long term improvement in renal function and prolongation of survival is timely liver transplantation. ${ }^{2}{ }^{3}$ However, most of these patients are nontransplant candidates at the time of HRS diagnosis but require intensive medical management which may include haemodialysis in some patients. ${ }^{4}$ Furthermore, transplant organ shortage limits urgent transplant even for transplantable patients.

Patients with refractory ascites who are at high risk of HRS can be effectively treated by insertion of a transjugular intrahepatic portosystemic stent-shunt (TIPS). However, data on recovery of renal function after TIPS in these patients are controversial. One study reported an increase in glomerular filtration rates (GFR) in six month survivors. ${ }^{5}$ In another small randomised trial comparing TIPS with large volume paracentesis for refractory ascites, GFR improved only marginally after TIPS while natriuresis increased significantly. ${ }^{6}$ As refractory ascites and HRS share a similar pathophysiology, ${ }^{2}$ TIPS has been tried as a rescue measure in patients with advanced HRS. So far, preliminary short term data are favourable. However, these series are small (1-7 severe HRS patients) and often lack follow up data beyond three months. ${ }^{7-14}$ Furthermore, HRS was often defined differently and included patients who were candidates for transplant rescue..$^{71012}$ This hampers outcome analysis, especially for those high risk cirrhotic patients who are actually not transplant candidates at the time of HRS diagnosis.

The present phase II study provides prospective long term data on HRS outcome following TIPS as rescue treatment in a large cohort of cirrhotics in whom liver transplantation was contraindicated at the time of HRS diagnosis.

Patients and methods

STUDY POPULATION AND DEFINITION OF HRS Between January 1995 and November 1998 we evaluated 45 consecutive patients with ad-

Abbreviations used in this paper: TIPS, transjugular intrahepatic portosystemic stent-shunt; HRS, hepatorenal syndrome; GFR, glomerular filtration rates; ET-1, endothelin 1; PRA, plasma renin activity; AR, active renin. 


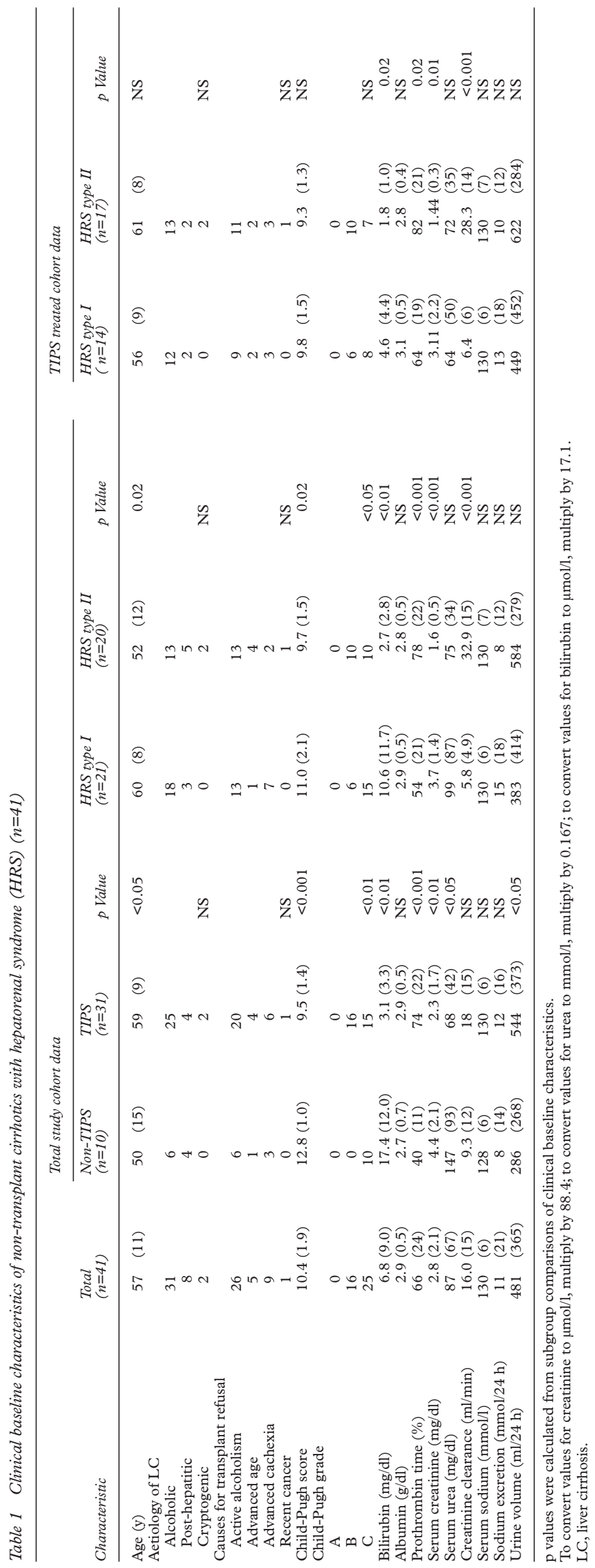

vanced cirrhosis of the liver, refractory ascites, and recently detected renal insufficiency. Forty one of these patients ( 25 males, 16 females) were excluded from liver transplantation as a therapeutic option and were enrolled in the present study of non-surgical management (table 1). The cause of liver cirrhosis was alcoholism in 31 patients and chronic viral hepatitis in eight. Two patients had cryptogenic cirrhosis. Within two months prior to detection of renal insufficiency, 22 patients $(54 \%)$ had suffered clinical complications related to portal hypertension, such as upper gastrointestinal haemorrhage (12 patients) and spontaneous bacterial peritonitis (10 patients). These complications had been effectively treated and none of these patients had a relapse within at least two weeks prior to diagnosis of renal failure. At presentation, 26 alcoholics were still active drinkers and nine of these had suffered recent severe alcohol abuse and clinically suspected concomitant acute alcoholic hepatitis (liver biopsy was performed in two and confirmed the clinical diagnosis). Thirty one patients were considered eligible for TIPS as an ultimate rescue treatment whereas 10 (25\%) were excluded from TIPS because of one or more persistent contraindications (bilirubin levels $\geqslant 15$ $\mathrm{mg} / \mathrm{dl}$ (five), Child-Pugh points $>12$ (four), or spontaneous severe encephalopathy (three)).

All patients were studied after a minimum of five days without diuretics on a $50 \mathrm{mmol} /$ day sodium diet as part of their oral nutrition. Oliguric patients had a bladder catheter inserted during diagnostic work up. Assessment of renal function was based on at least 2-3 samples of daily urine volume and serum parameters obtained prior to individual treatment assignment. All patients fulfilled recent consensus criteria for $\mathrm{HRS},{ }^{2}$ which are, in brief: proven liver cirrhosis with severe ascites; no recent nephrotoxic medication or severe fluid losses; no renal abnormalities on ultrasound; no nephritic sediment; proteinuria less than $500 \mathrm{mg} /$ day; no signs of active infection or other reasons for renal impairment; and finally no improvement in renal function after withdrawal of diuretics and appropriate volume expansion ( $200 \mathrm{ml}$ of $20 \%$ albumin and/or 1-2 litres of isotonic saline). HRS was graded as HRS type I (severe and rapidly progressive: serum creatinine $>2.5 \mathrm{mg} / \mathrm{dl}$ or creatinine clearance $<20 \mathrm{ml} / \mathrm{min} ;>50 \%$ loss in clearance within two weeks) or HRS type II (moderate and stable or slowly progressive: serum creatinine $>1.5 \mathrm{mg} / \mathrm{dl}$ or creatinine clearance $<40$ $\mathrm{ml} / \mathrm{min}$ ). Overall, we diagnosed 21 patients with type I and 20 with type II HRS. Fourteen patients with type I HRS (seven of whom required haemodialysis due to persistent oligoanuria with life threatening pulmonary oedema and hyperkalaemia) and 17 with type II HRS received TIPS treatment. The median interval from the first detection of renal insufficiency to TIPS insertion was shorter in type I HRS (2.2 weeks; range 0.3-6) than in type II HRS (4.0 weeks; range $1.5-5$ ) but mean values were not significantly different (3.0 (1.6) v 4.3 (0.3) weeks). Among the 10 non-stented patients, seven had type I and three had type II 
HRS. Preliminary short term data on changes in renal function up to six months after TIPS treatment in 16 of our patients (six with type I and 10 with type II HRS) have been reported previously. ${ }^{13}$

All patients were considered ineligible for liver transplantation for the following reasons (table 1): active alcoholism $(n=26)$, ineligibility for major surgery (age over 65 years or advanced cachexia) $(n=14)$, and recent oropharyngeal cancer $(n=1)$. Six active alcoholics and four patients considered poor candidates for major surgery were excluded from TIPS and received the best medical support, including adequate volume/electrolyte management, paracentesis and albumin substitution, and vasoconstrictive drugs, as indicated. ${ }^{2}$ Patients gave written informed consent for TIPS as a salvage procedure as well as for portal and systemic blood sampling according to the study protocol which was approved by the local ethics committee.

\section{TIPS INSERTION}

The day before the scheduled TIPS insertion all patients received a therapeutic paracentesis followed by intravenous administration of albumin (8 $\mathrm{g} / 1$ ascites volume). After an overnight fast and bed rest, patients were taken to the radiology department for the TIPS procedure. We punctured the portal system under ultrasound guidance using a 9 French liver biopsy needle (Cook, Germany) and established small diameter $(8-10 \mathrm{~mm})$ stent-shunts either with Palmaz stents or Wallstents, as described in other TIPS reports. ${ }^{15-17}$ Our target was a reduction in portal pressure gradient (portal minus vena cava superior pressure) of $30-50 \%$. In patients with no or only mild ascites after paracentesis this gradient differs only minimally from the more commonly assessed gradient of portal minus vena cava inferior pressure. ${ }^{18}$ For technical reasons, to minimise the amount of contrast media as well as the duration of the procedure, we used the modified gradient to assess the relative pressure changes induced by TIPS. The initial technical success rate was $100 \%$ without apparent adverse effects during the TIPS procedure. Patients received a single dose of antibiotic prophylaxis of cefuroxime (1.5 g), and midazolam $(5-15 \mathrm{mg})$ with pethidine $(75-150 \mathrm{mg})$ for anaesthesia. Intravenous heparin was given for prevention of shunt thrombosis (bolus dose of 2500-5000 U followed by constant infusion for 1-2 weeks, targeted at an activated partial thromboplastin time of $60-80$ seconds). TIPS patency was monitored by repeated Doppler ultrasound (twice weekly within the first two weeks) and routine radiography 10-17 days after TIPS in all but two patients. Thereafter, Doppler ultrasound was performed every 2-3 months or whenever clinically indicated. Additional radiography was performed only for suspected TIPS dysfunction. TIPS patency was maintained during the study period in all patients. Following a minimum of one week after TIPS, diuretics were gradually reintroduced (spironolactone $50-200 \mathrm{mg} /$ day and frusemide $20-80 \mathrm{mg} /$ day) if daily spontaneous excretion had doubled and/or exceeded $10 \mathrm{mmol} /$ day. Vasoactive drugs were not administered.

FOLLOW UP

Renal function parameters (serum creatinine, serum urea, creatinine clearance, urine volume, sodium excretion/ $\mathrm{UNaV}$ ) were assessed before and at $1,2,4,8,12-13$, and 24-26 weeks, and then every $2-3$ months after the TIPS procedure. Overall mean follow up time was 116 weeks (median 104; range 8-212). TIPS patients were followed for at least five months or until death and their mean follow up period was 128 weeks (median 126; range 22-212).

\section{DETERMINATION OF VASOCONSTRICTIVE}

MEDIATORS

We collected plasma (EDTA) from the last 20 consecutive TIPS patients (11 type I and nine type II HRS) from the portal vein (prior to stenting) and from the systemic circulation to determine endothelin-1 (ET-1, ELISA assay, reference values $0.73(0.03) \mathrm{pmol} / \mathrm{l})$ levels and activation of the renin-angiotensin system. The latter was estimated by measuring plasma renin activity (PRA) in 12 (RIA assay, reference values: baseline $<5 \mathrm{ng} / \mathrm{ml} / 3$ hours, stimulated $<10 \mathrm{ng} / \mathrm{ml} / 3$ hours) or by active renin (AR; RIA assay, reference values: baseline $<25 \mathrm{ng} / \mathrm{l}$, stimulated $<50 \mathrm{ng} / \mathrm{l}$ ) in the last eight patients. A second pair of portal and systemic plasma samples were obtained during the first invasive TIPS evaluation after $12-15$ days.

\section{STATISTICAL ANALYSIS}

Follow up parameters were compared with baseline values using two sided Student's $t$ tests or by Mann-Whitney U test, as appropriate, for patients at risk at a given time interval after confirmation of changes over time by MANOVA. Categorical data were analysed with Fisher's exact test. All values are given as mean (SD). Differences at $\mathrm{p}<0.05$ were considered significant. Estimation of survival was based on the Kaplan-Meier method and on the log rank test for group comparisons. Cox regression models were used for multivariate analysis of outcome predictors. SPSS-PC+ software was used on an IBM computer.

\section{Results}

STUDY POPULATION

Baseline clinical characteristics in the total study population (table 1 ) were similar for type I and type II HRS patients with respect to age and aetiology of liver cirrhosis, but type I HRS patients showed a more advanced degree of liver dysfunction, as assessed by routine parameters. Interestingly, despite significantly different glomerular filtration rate parameters, there was a similar reduction in serum sodium and sodium excretion in type I and type II patients.

RENAL FUNCTION AFTER TIPS INSERTION Thirty one patients received TIPS (table 1 ) on average 3.4 (1.3) weeks after detection of renal insufficiency, which reduced the portal pres- 
sure gradient from 21 (4) to 13 (5) $\mathrm{mm} \mathrm{Hg}$ $(\mathrm{p}<0.001)$. Within the first month after TIPS, we observed marked improvements in all renal function parameters (MANOVA, $\mathrm{p}<0.01$ ) whereas parameters of liver function showed a transient deterioration (table 2). Thereafter, renal function showed sustained stabilisation (fig 1) under careful reintroduction of diuretics (100-200 mg of spironolactone and $40-80 \mathrm{mg}$ of frusemide per day). There was a marked and significant spontaneous increase in urinary sodium excretion by one week after TIPS insertion when patients were not receiving diuretics (fig 1, table 2). Interestingly, it was possible to withdraw haemodialysis in four of seven patients (days 12, 14, 18, and 22). These patients survived at least 10 months with compensated renal function (serum creatinine 2.1, $1.9,1.8$, and $1.6 \mathrm{mg} / \mathrm{dl}$ ). Parallel with increased sodium excretion ( $>50 \mathrm{mmol} / 24$ hours), ascites improved in 14 patients and completely resolved in 10 . However, seven of 31 patients $(23 \%)$ did not respond and still had refractory ascites needing paracentesis after one month. All but one died within three months after TIPS.

With respect to non-TIPS patients, renal function worsened progressively in all but one patient. One young man with histological signs of acute alcohol hepatitis and chronic liver cirrhosis experienced gradual recovery from type I to type II HRS. He was the only non-TIPS patient who survived more than three months after HRS onset.
SHUNT PATENCY AND ADVERSE EVENTS AFTER TIPS

During the first year of follow up we observed major shunt stenosis in six patients and complete shunt occlusion in one man. TIPS function was successfully re-established by balloon dilatation (three) or by stent prolongation (three) in six patients, while in one patient lethal sepsis excluded reintervention at the time of diagnosis of TIPS dysfunction.

Within four weeks after TIPS, patients experienced on average a mild transient deterioration in some liver function parameters (table 2). During the first three months after TIPS we observed de novo severe encephalopathy in three patients or deterioration in pre-existing mild to moderate encephalopathy in eight patients. Six of these patients had limited short term episodes managed by lactulose but five experienced progressive liver failure. Of note, six of these 11 patients had continued alcohol abuse after TIPS and hospital release.

\section{VASOCONSTRICTIVE MEDIATORS}

Mean baseline systemic ET-1 was elevated 2-3-fold above the upper normal limit and remained unchanged after TIPS. Baseline portal ET-1 was higher than ET-1 in the peripheral systemic circulation and decreased significantly after TIPS (table 2). In contrast, portal and systemic activation of the renin-angiotensin system (estimated by PRA and AR) were similar and both decreased from an eightfold elevation above normal to a 2-3-fold elevation two

Table 2 Liver and renal function, systemic circulation, and vasoactive mediators during the first weeks after transjugular intrahepatic portosystemic stent-shunt (TIPS) insertion

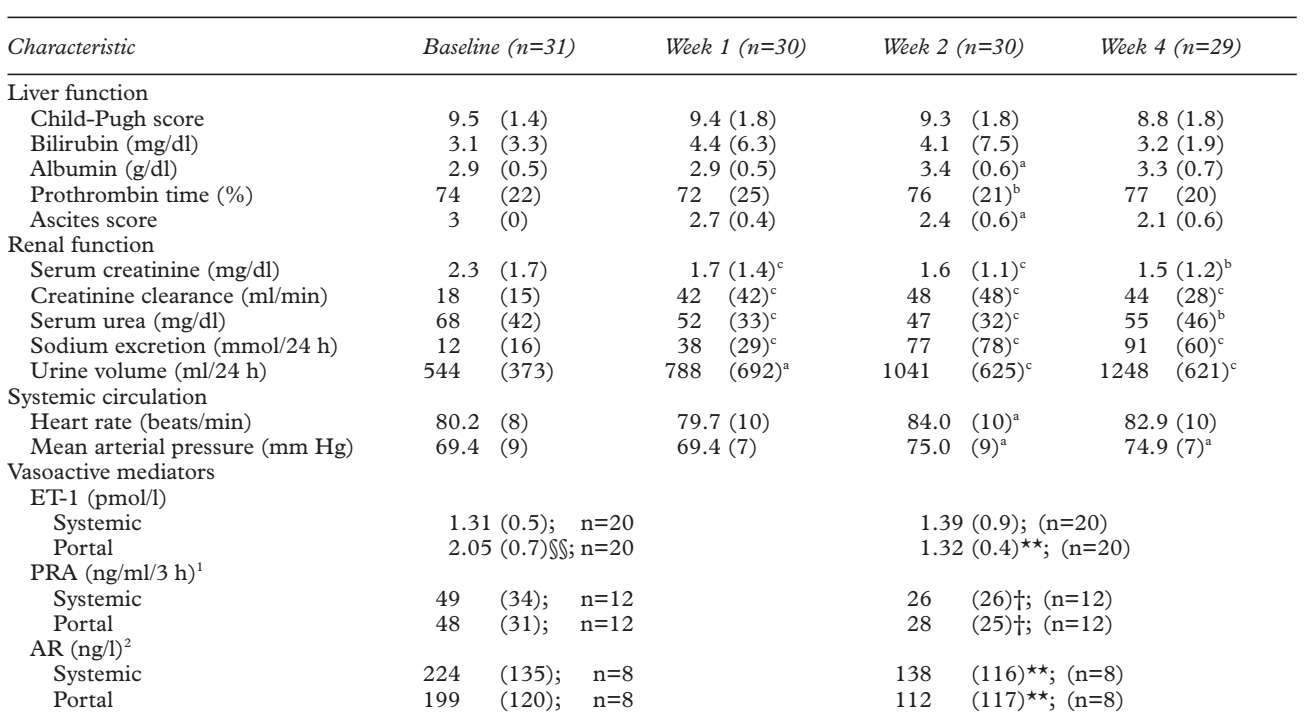

Parameters (mean (SD)) of liver and renal function, and systemic circulation (heart rate, mean arterial pressure (MAP)) before and up to four weeks after TIPS.

Baseline endothelin-1 (ET-1) and renin-angiotensin system activation (estimated by plasma renin activity (PRA) or active renin $(\mathrm{AR})$ ) in the systemic venous and portal circulation were determined in the last consecutive 24 patients and repeated two weeks after TIPS in 20 patients.

Within four weeks, MANOVA detected significant improvements in all renal function parameters, ascites score, and MAP, whereas changes in biochemical liver function parameters showed only a trend towards transient deterioration. Differences over time versus baseline were compared for renal function parameters, ascites score, and MAP for one, two, and four week intervals, whereas all other parameters were tested once for the two week interval.

Differences in liver and renal function $v$ baseline: ${ }^{\mathrm{a}} \mathrm{p}<0.05,{ }^{\mathrm{b}} \mathrm{p}<0.01,{ }^{\mathrm{c}} \mathrm{p}<0.001$.

Vasoactive mediators: ${ }^{1}$ PRA: baseline $v$ control $(\mathrm{n}=12) ;{ }^{2} \mathrm{AR}$ : baseline $v$ control $(\mathrm{n}=8)$

\\Portal $v$ systemic ET-1 baseline levels, $\mathrm{p}<0.01$; †control $v$ baseline, $\mathrm{p}<0.05$; ${ }^{\star \star}$ control $v$ baseline, $\mathrm{p}<0.01$. 

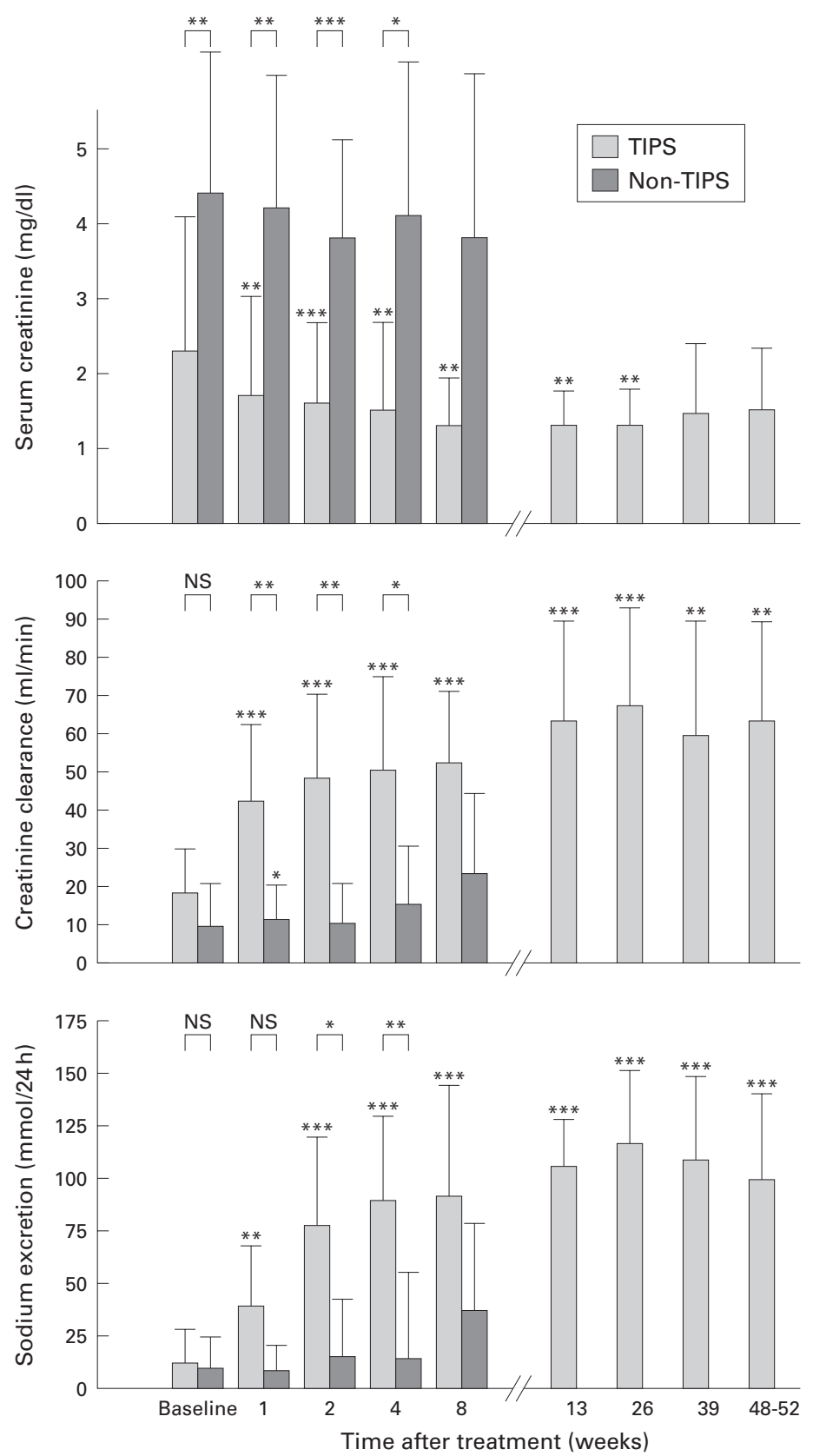

TIPS patients: $\quad n=31 n=30 n=30 n=29 n=26 \quad n=24 n=20 n=15 n=12$ Non-TIPS patients: $n=10 \quad n=10 \quad n=5 \quad n=3 \quad n=2 \quad n=1 \quad n=1 \quad n=1 \quad n=1$

Figure 1 Mean (SD) serum creatinine levels ( $m g / d l$; to convert values to $\mu$ mol/l multiply by 88.4), creatinine clearance ( $\mathrm{ml} / \mathrm{min})$, and sodium excretion ( $\mathrm{mmol} / 24$ hours) up to one year after diagnosis of hepatorenal syndrome in 41 patients of whom 31 received a transjugular intrahepatic portosystemic stent-shunt (TIPS) and 10 were excluded from receiving TIPS. $p$ values were calculated using the Mann-Whitney $U$ test and represent comparisons with baseline for patients at risk or between treatment groups at given time intervals: ${ }^{\star} p<0.05,{ }^{\star *} p<0.01,{ }^{\star *} p<0.001$. Note that all values prior to and up to one week after TIPS were determined without diuretics. Thereafter, moderate diuretic medication with furosemide (40-80 mg/day) and spironolactone (100-200 mg/day) was reintroduced and adapted to the patients' needs.

weeks after TIPS, despite reintroducing diuretic therapy (table 2).

\section{SURVIVAL ANALYSIS}

Survival rates (Kaplan-Meier) of the total cohort were $63 \%, 56 \%, 39 \%$, and $29 \%$ after three, six, 12, and 18 months, respectively, with a mean survival of 75 (14) weeks (95\% CI 48-102, median 34). TIPS patients showed significantly better survival than non-TIPS patients with more advanced liver dysfunction at baseline (log rank 18.3; p<0.001) (fig 2A, 2D). After TIPS, three, six, 12, and 18 month survival rates were $81 \%, 71 \%, 48 \%$, and $35 \%$ (mean survival 92 (16) weeks; 95\% CI 60-123; median 49) compared with non-TIPS patients (three month survival of $10 \%$ and a mean survival of 12 (8.5) weeks (95\% CI 0.01-28; median 2.0)). Type II HRS patients had a better chance of survival than type I patients (log rank 5.04; $p=0.025$ ) (fig 2B). TIPS patients whose ascites were mobilised within one month (clinical responder) survived longer than patients who did not respond (log rank 46.6; $\mathrm{p}<0.001$ ) (fig 2C). Interestingly, even type I patients treated with TIPS $(n=14)$ achieved three, six, and 12 month survival rates of $64 \%$, $50 \%$, and $20 \%$, which were significantly better than the survival rates of type I patients who did not undergo TIPS $(\mathrm{n}=7 ; \mathrm{p}<0.01)$ with similar renal dysfunction at baseline (fig 2D; direct Kaplan-Meier comparison not plotted). Combined survival analysis of all 21 type I HRS patients showed three, six, and 12 month survival rates of $48 \%, 38 \%$, and $16 \%$ (KaplanMeier plot not shown).

For the TIPS patients, multivariate Cox regression analysis, including baseline factors that were univariately related to death (bilirubin, prothrombin time, Child-Pugh, type of HRS), revealed only bilirubin $(p<0.001)$ and type of HRS $(p<0.05)$ as independent survival predictors. Most deaths after TIPS (83\%) were related to progressive liver failure. One patient suffered a procedure related fatal liver haemorrhage and two TIPS patients died from non-liver related events (week 22, cancer progress; week 45 , sepsis caused by colon perforation as diagnosed at autopsy). Subgroup survival analysis in TIPS patients showed that three month survivors had better baseline liver function (bilirubin 1.7 (1) v 8.7 (5.3) $\mathrm{mg} / \mathrm{dl}$, $\mathrm{p}<0.001$; Child-Pugh score 9.2 (1.3) $v 10.8$ (1), $\mathrm{p}=0.08$ ) but similar renal dysfunction (creatinine clearance 20 (12) $v 18(15) \mathrm{ml} / \mathrm{min}$, NS) compared with non-survivors.

\section{TRANSPLANT REEVALUATION}

Transplant re-evaluation in three month survivors revealed that 10 of 16 initially active alcoholics still abused alcohol after successful TIPS treatment. Six of these 10 persistent alcoholics died within one year after TIPS. In the remaining six, three month survivors (five after TIPS, one without TIPS) who had stopped drinking, liver function improved gradually after 2-3 months so that transplantation was not necessary within the first six months of our study period. Two of these patients who abstained were successfully transplanted seven months and two years after TIPS with stable renal function (serum creatinine 1.0 and $1.8 \mathrm{mg} / \mathrm{dl}$, no ascites).

\section{Discussion}

In this phase II study, we have reported on a larger cohort of non-transplantable cirrhotics with HRS diagnosed according to recent consensus criteria. The median follow up of two years after HRS diagnosis provides the 

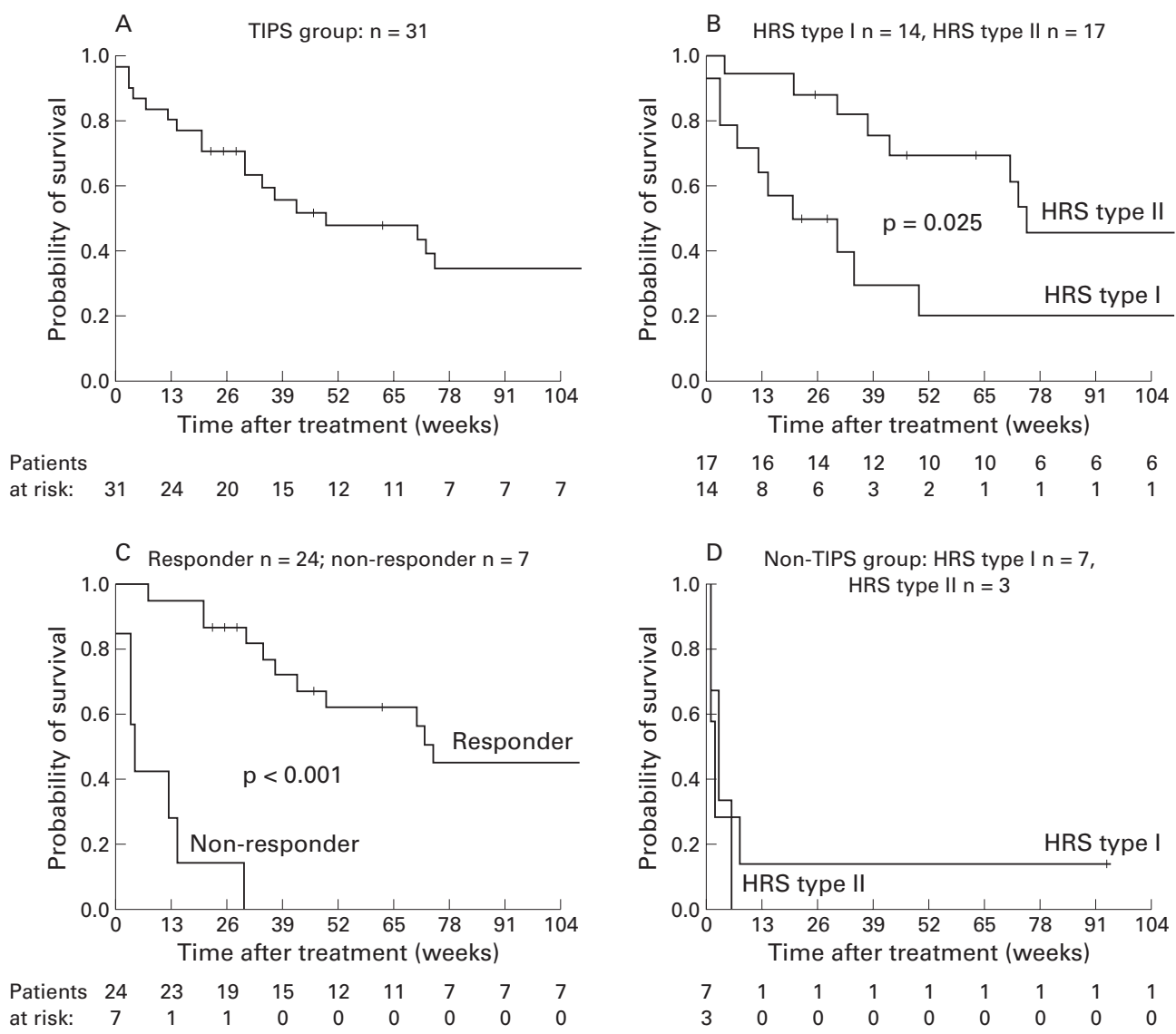

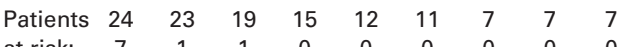

Figure 2 Kaplan-Meier survival analysis. (A) Cohort of patients with hepatorenal syndrome (HRS) treated by transjugular intrahepatic portosystemic stent-shunt (TIPS) $(n=31)$; (B) survival analysis after TIPS according to HRS subtypes at baseline; (C) survival analysis according to clinical response (improved sodium excretion and ascites control within one month) or no response after TIPS; (D) survival analysis of non-TIPS patients ( $n=10$ : type I HRS, $n=7 ;$ type II HRS, $n=3$ ) receiving the best medical support. $p$ values were derived from subgroup comparisons using the log rank test.

longest HRS outcome analysis apart from post-transplant series. ${ }^{23}$ The majority of our cohort experienced sustained improvement in kidney function together with a remarkably high overall one year survival rate of almost $40 \%$. We attribute this benefit to the fact that we considered about $75 \%$ of our patients eligible for TIPS as rescue treatment, of whom $77 \%(24 / 31)$ (that is, $59 \%$ of the total cohort) exhibited rapid and sustained improvement in GFR and sodium excretion. This response allowed reintroduction of mild diuretic therapy and even withdrawal from haemodialysis in four of seven patients. However, $25 \%$ of our high risk patients were excluded from undergoing TIPS because of poor liver function, and almost $25 \%$ of TIPS patients did not respond. Both of the latter groups showed a poor prognosis (fig 2C, 2D) similar to previous reports, ${ }^{1}$ with a median survival of approximately 2 weeks. As our total cohort was comparable with earlier large HRS series ${ }^{134}$ with respect to renal and liver function, it is unlikely that the more favourable outcome resulted primarily from patient selection. In addition, even overall survival in the subgroup of our 21 type I HRS patients (14 treated by TIPS) was remarkably high, with three and six month survival rates of $48 \%$ and $38 \%$ compared with the largest series of HRS patients ${ }^{1}$ (in which probably most but not all had type I HRS) which showed a three month survival rate of $9 \%$ under medical treat- ment (long term survival rates were not given; median survival 1.7 weeks). Thus it is likely that former HRS series probably included a high proportion of patients who today might benefit from TIPS as a salvage measure.

Although liver transplantation is the treatment of choice for cirrhotic patients with HRS, less invasive approaches have been evaluated because many of these patients are nontransplant candidates (for example due to advanced age, cachexia, and/or active alcoholism). Alternative non-surgical medical approaches using prostaglandins, vasopressin analogues, dopamine, octreotide, or $\mathrm{N}$-acetylcysteine are based mainly on short term applications in small series. ${ }^{219-26}$ Data on vasoactive drugs used as a bridge to transplantation are promising but still unclear. ${ }^{27}{ }^{28}$ Long term administration of vasopressin analogues appears to be effective but is often contraindicated or limited by severe vasoconstrictive side effects. ${ }^{20-23}$

TIPS is a more recent option for the treatment of HRS by semi-invasive portal decompression. Compared with other series, our study provides long term data based on the largest cohort of cirrhotics with HRS diagnosed as type I or type II according to current consensus criteria. ${ }^{2}$ Of note is the fact that outcome was not influenced by timely transplant rescue within the first critical months after HRS diagnosis, as at baseline all patients were 
non-transplant candidates. It is well known that portal decompressive surgery (that is, a portacaval end-to-side anastomosis or a sideto-side shunt) not only prevents recurrent bleeding but also prevents the formation of ascites in a larger number of patients than therapies that do not lower portal hypertension. ${ }^{29}$ As HRS is the extreme form of refractory ascites, it is sensible to treat HRS with TIPS, especially as this procedure does not carry the risk of open surgery.

Timely TIPS insertion is probably crucial for successful HRS management. Based on our data, recovery was still possible 4-6 weeks even after type I HRS onset in anuric patients who were bridged to TIPS by haemodialysis. However, a clear time limit for TIPS has yet to be defined. As all HRS patients have severe ascites and suffer spontaneously reduced food consumption, chronic fatigue with prolonged bed rest, and progressive muscle atrophy, serum creatinine and urea levels are low in relation to endogenous creatinine clearance data (tables 1 , 2). This discrepancy may lead to diagnostic overestimation of GFR with delayed HRS diagnosis.

The pathogenesis of HRS is still incompletely understood. ${ }^{2} 2{ }^{28}{ }^{30-35}$ Cirrhotic patients with HRS suffer from peripheral and splanchnic vasodilatation combined with severe renal vasoconstriction mediated by several vasoconstrictive factors such as renin, catecholamines, endothelins, and prostaglandin derivatives. ${ }^{35-39}$ In this situation TIPS insertion not only leads to portal decompression but also to better refilling of the central venous system, although the peripheral hyperdynamic status persists. ${ }^{39-41}$ Improved refilling can decrease several vasoconstrictors and thus contribute to better renal perfusion leading to an increase in GFR and sodium excretion. ${ }^{39}$ These considerations are supported by the observed lesser activation of the renin-angiotensin system after TIPS insertion in our patients despite reintroduction of diuretics, which probably blunts further renin decline to a certain degree. Beneficial short term effects of TIPS on renin activity have been reported in patients with refractory ascites $^{39-41}$ and with HRS. ${ }^{14}$ Interestingly, we detected a significant reduction in portal ET-1 levels, while elevated systemic ET-1 levels remained unchanged after TIPS. Similar differences in ET-1 levels between the portal and renal compartment have been described in cirrhotics with ascites after TIPS. ${ }^{42}$ Both observations indicate that local or paracrine ET-1 may be more relevant for kidney dysfunction in HRS than circulating ET-1. ${ }^{14}{ }^{23}{ }^{42-45}$ Furthermore, a decrease in sinusoidal pressure after TIPS may reduce renal sodium retention of the proximal tubule mediated by a sympathetic nervous pathway. Indeed, animal experiments suggest such a hepatorenal reflex, as an acute rise in sinusoidal pressure caused immediate sodium retention. ${ }^{374}$ Also, in humans, an increase in short term portal pressure induced an immediate reduction in renal perfusion. ${ }^{47}$

Despite potential benefits in renal function, TIPS affects portal venous liver perfusion and thereby carries the risk of progressive liver fail- ure. Therefore, we restricted its placement to patients whose livers were considered to have enough residual capacity to counterbalance reduced perfusion. We excluded patients with Child-Pugh scores $>12$, bilirubin levels $\geqslant 15$ $\mathrm{mg} / \mathrm{dl}$, or those with severe spontaneous encephalopathy. We are aware that these criteria have been arbitrarily set and that the bilirubin limit in particular was quite generous but reasonable for the given desperate therapeutic situation. Regarding the Child-Pugh score criteria, another report applied similar restrictions. ${ }^{14}$ However, we need more controlled data to improve selection regarding the degree of liver dysfunction. In our series early mortality in patients with high baseline bilirubin levels was still substantial after TIPS. This result is similar to outcome data in other reports on shunting or endoscopic treatments for portal hypertension. ${ }^{15-1748}$ Thus prior to TIPS treatment, bilirubin should preferably be stable below $5 \mathrm{mg} / \mathrm{dl}$. We are aware that this criterion probably excludes many cirrhotic patients with HRS and concomitant acute alcoholic hepatitis from early TIPS. However, these patients may become TIPS candidates when HRS persists even after recovery of liver function under strict alcohol abstinence. Such a consideration encourages the option of transient dialysis treatment, at least in some patients. In the majority of our stented patients with stable bilirubin levels prior to TIPS we observed only mild and transient deteriorations in liver function applying small diameter shunts with limited initial portal decompression (35-45\%, measured for technical reasons as reduction in portal vein to vena cava superior pressure gradient). Compared with initial mean portal pressure gradient reductions of approximately $50-60 \%$ reported in

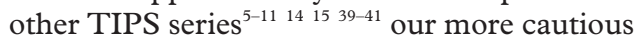
portal decompression together with our selection criteria for TIPS treatment may have contributed to the rather favourable long term outcome of our total HRS cohort.

In conclusion, limited portal decompression by TIPS represents a promising new option for treating selected HRS patients with sustained efficacy, even without liver transplantation, and warrants further prospective controlled trials in non-transplant and even in transplant candidates. We believe that our long term data provide an adequate basis to plan such controlled trials on the role of TIPS in the management of HRS.

The results of this work were presented in part at the 47 th Annual Meeting of the American Association for the Study of Liver Diseases held in Chicago (Hepatology 1996;24:A1292) and preliminary six month renal function data appeared as a research letter in The Lancet (1997;349:697-8)

This work was supported by a grant from the Lucie Bolte Foundation. We would like to thank D Bammer, R Sunkel, $\mathrm{H}$ Maar-Schnabel, and A Bäcker for excellent technical assistance, Dr M Malek for final figure layout, and S Körner for secretarial work.

1 Gines A, Escorsell A, Gines P, et al. Incidence, predictive factors, and prognosis of the hepatorenal syndrome in cirrhosis with ascites. Gastroenterology 1993;105:229-36.

2 Arroyo V, Gines P, Gerbes AL, et al. Definition and diagnostic criteria of refractory ascites and hepatorenal diagnostic criteria of refractory ascites and hepa
syndrome in cirrhosis. Hepatology 1996;23:164-76.

3 Gonwa TA, Morris CA, Goldstein RM, Husberg BS, Klintmalm GB. Long-term survival and renal function following 
liver transplantation in patients with and without hepatorenal syndrome- experience in 300 patients. Transplantation nal syndrome- -

4 Keller F, Heinze H, Jochimsen F, Paßfall J, Schuppan D, Büttner P. Risk factors and outcome of 107 patients with decompensated liver disease and acute renal failure (including 26 patients with hepatorenal syndrome): The role of hemodialysis. Ren Fail 1995;17:135-46.

5 Ochs A, Rössle M, Haag K, et al. The transjugular intrahepatic portosystemic stent-shunt procedure for refractory ascites. N Engl F Med 1995;332:1192-7.

6 Lebrec D, Giuily N, Hadengue A, et al. Transjugular intrahepatic portosystemic shunts: comparison with paracentesis in patients with cirrhosis and refractory ascites: a randomized trial. F Hepatol 1996;25:135-44.

7 Lake JR, Ring E, LaBerge JM, Gordon R, Roberts J, Ascher $\mathrm{N}$. Transjugular intrahepatic portacaval stent shunts in patients with renal insufficiency. Transplant Proc 1993;25: patients

8 Ochs A, Rössle M, Haag K, et al. TIPS for hepatorenal syndrome. Hepatology 1994;20(Pt 2):114A.

9 Spahr L, Fenyves D, N'Guyen VV, et al. Improvement of hepatorenal syndrome by transjugular intrahepatic portosystemic shunt. Am Gastroente

10 Lerut J, Goffette P, Laterre PF, Donataccio M, Reynaert MS, Otte JB. Sequential treatment of hepatorenal syndrome and posthepatic cirrhosis by intrahepatic portosystemic shunt (TIPSS) and liver transplantation. Hepatogastroenterology 1995;42:985-7.

11 Sturgis TM. Hepatorenal syndrome: resolution after transjugular intrahepatic portosystemic shunt. $\mathcal{f}$ Clin Gastroenterol 1995;20:241-3.

12 Alam I, Bass NM, LaBerge JM, Ring E, Somberg KA. Treatment of hepatorenal syndrome with the transjugular intrahepatic shunt (TIPS), Gastroenterology $1995 ; 108$ : A1024.

13 Brensing KA, Textor J, Strunk H, Klehr HU, Schild H, Sauerbruch $\mathrm{T}$. Transjugular intrahepatic portosystemic stenterbruch T. Transjugular intrahepatic portosystemic stent-

14 Guevara M, Gines P, Bandi JC, et al. Transjugular intrahepatic portosystemic shunt in hepatorenal syndrome: Effects patic portosystemic shunt in hepatorenal syndrome: Effects
on renal function and vasoactive systems. Hepatology 1998; 28:416-22.

15 Rössle M, Haag K, Ochs A, et al. The transjugular intrahepatic portosystemic stent-shunt procedure for variceal bleeding. N Engl F Med 1994;330:165-71.

16 Conn HO. Transjugular intrahepatic portal-systemic shunts: The state of the art. Hepatology 1993;17:148-58.

17 Stanley AJ, Jalan R, Forrest EH, Hayes PC. Longterm follow up of transjugular intrahepatic portosystemic stent shunt (TIPSS) for the treatment of portal hypertension: results in 130 patients. Gut 1996;39:479-85.

18 Brensing KA, Neubrand M, Textor J, et al. Endoscopic manometry of esophageal varices: evaluation of a balloon technique compared with direct portal pressure measurement. f Hepatol 1998;29:94-102.

19 Fevery J, Van Cutsem E, Nevens F, Van Steenbergen W, Verbeckmoes R, DeGroote J. Reversal of hepatorenal syndrome in four patients by peroral misoprostol (prostasyndrome in four patients by peroral misoprostol (prostaglandin E1 analogue)

20 Lenz K, Hörtnagel H, Druml W, et al. Ornipressin in the treatment of functional renal failure in decompensated liver cirrhosis. Effects on renal hemodynamics and atrial natriuretic factor. Gastroenterology 1991;101:1060-7.

21 Salo J, Gines A, Quer JC, et al. Renal and neurohormonal changes following simultaneous administration of systemic vasoconstrictors and dopamine or prostacyclin in cirrhotic patients with hepatorenal syndrome. $\mathcal{F}$ Hepatol 1996;25: 916-23.

22 Cervoni JP, Lecomte T, Cellier C, et al. Terlipressin may influence the outcome of hepatorenal syndrome complicating alcoholic hepatitis. Am f Gastroenterol 1997;92:211314.

23 Guevara M, Gines P, Fernandez-Esparrach G, et al. Reversibility of hepatorenal syndrome by prolonged administration of ornipressin and plasma volume expansion. Hepatology 1998;27:35-41.

24 Kaffy F, Borderie C, Chagneau C, et al. Octreotide in the treatment of hepatorenal syndrome in cirrhotic patients. $\mathscr{f}$ Hepatol 1999;30:174.
25 Holt S, Goodier D, Marley R, et al. Improvement in renal function in hepatorenal syndrome with $\mathrm{N}$-acetylcysteine. Lance 1999:353:294-5.

26 Angeli P, Volpin R, Gerunda G, et al. Reversal of type 1 hepatorenal syndrome with administration of midodrine and octreotide. Hepatology 1999;29:1690-7.

27 Wong F, Blendis L. Therapy for hepatorenal syndrome. Gastroenterology 1998;115:503-4.

28 LeMoine O. Hepatorenal syndrome-outcome after liver transplantation. Nephrol Dial Transplant 1998;13:20-2.

29 Castells A, Salo J, Planas R, et al. Impact of shunt surgery for variceal bleeding in the natural history of ascites in cirrhosis: a retrospective study. Hepatology 1994;20:58491.

30 Epstein M. Hepatorenal syndrome. In: Epstein M, ed. The kidney in liver disease, 4th edn. Philadelphia: Hanley and Belfus, 1996:75-108.

31 Brensing KA, Woitas R, Heller J, et al. Significantly impaired glomerular perfusion and filtration rate already in patients glomerular perfusion and filtration rate already in patients
with compensated liver cirrhosis. Gastroenterology 1996; with comp

32 Henriksen JH. Cirrhosis: Ascites and hepatorenal syndrome. Recent advances in pathogenesis. If Hepatol 1995;23 (suppl 1):25-30.

33 Bataller R, Gines P, Guevara M, Arroyo V. Hepatorenal syndrome. Semin Liver Dis 1997;17:233-47.

34 Koppel MH, Cohburn JW, Mims MM, Goldstein H, Boyle JD, Rubini ME. Transplantation of cadaveric kidneys from patients with hepatorenal syndrome: evidence for the functional nature of renal failure in advanced liver disease. $N$ Engl f Med 1969;280:1367-71.

35 Epstein $M$. The hepatorenal syndrome-newer perspectives. N Engl f Med 1992;327:1810-11.

36 Moore K, Wendon J, Frazer M, Karani J, Williams R, Badr K. Plasma endothelin immunoreactivity in liver disease and hepatorenal syndrome. N Engl F Med 1992;327:1774-8.

37 Henriksen JH, Ring-Larsen H. Hepatorenal disorders: role of sympathetic nervous system. Semin Liver Dis 1994;14: of symp. 43 .

38 Sacerdoti D, Balazy M, Angeli P, Gatta A, McGiff JC. Eicosanoid excretion in hepatic cirrhosis. Predominance of 20-HETE. F Clin Invest 1997;100:1264-70.

39 Wong F, Sniderman K, Liu P, Allidina Y, Sherman M, Blendis L. Transjugular intrahepatic portosystemic stent shunt: effects on hemodynamics and sodium homeostasis in cirrhosis and refractory ascites. Ann Intern Med 1995;122:816-22.

40 Quiroga J, Sangro B, Nunez M, et al. Transjugular intrahepatic portal-systemic shunt in the treatment of refractory ascites: effect on clinical, renal, humoral, and hemodynamic parameters. Hepatology 1995;21:986-94.

41 Somberg KA, Lake JR, Tomlanovich SJ, LaBerge JM, Feldstein V, Bass NM. Transjugular intrahepatic portosystemic shunts for refractory ascites: assessment of clinical and hormonal response and renal function. Hepatology 1995;21: 709-16.

42 Martinet JP, Legault L, Cernacek P, et al. Changes in plasma endothelin-1 and big endothelin-1 induced by transjugular intrahepatic portosystemic shunts in patients with cirrhosis and refractory ascites. F Hepatol 1996;25:700-6.

43 Gerbes AL, Gulberg V, Waggershauser T, Holl J, Reiser M. Renal effects of transjugular intrahepatic portosystemic shunt in cirrhosis: Comparison of patients with ascites, with refractory ascites, or without ascites. Hepatology 1998; 28:683-8.

44 Gerbes AL, Moller S, Gulberg V, Henriksen JH. Endothelin-1 and -3 plasma concentration in patients with cirrhosis: role of splanchnic and renal passage and liver function. Hepatology 1995;21:735-9.

45 Epstein M, Goligorsky MS. Endothelin and nitric oxide in hepatorenal syndrome: a balance reset. 7 Nephrol 1997;10: 120-35.

46 Lang F, Tschernko E, Schulze E, et al. Hepatorenal reflex regulating kidney function. Hepatology 1991;14:590-4.

47 Jalan R, Forrest EH, Redhead DN, Dillon JH, Hayes PC. Reduction in renal blood flow following acute increase in the portal pressure: evidence for the existence of a hepatorenal reflex in man? Gut 1997;50:664-70.

48 D'Amico G, Pagliaro L, Bosch J. The treatment of portal hypertension: a meta-analytic review. Hepatology 1995;22: $332-54$ 